\title{
Apparent diffusion coefficient for differentiating between benign and malignant hepatic focal lesions
}

\author{
Sherif Abugamra', Aya Yassin ${ }^{1}$, Asmaa Saber Mostafa Abdel-Rehim² and Dina Sayed Sheha ${ }^{2 *}$ (D)
}

\begin{abstract}
Background: The aim of this study was to prospectively evaluate the role of diffusion weight MRI (DWI) in the characterization of hepatic focal lesions by using apparent diffusion coefficient (ADC). Thirty patients (18 women, 12 men; mean age 48.5 years) with hepatic focal lesions were included in this study. Patients underwent DW MR imaging with the SPLICE sequence. ADC of each focal lesion carcinoma was calculated from DW MR Images obtained with low and high $b$ values. ADCs were compared among pathological types of focal lesions.

Results: Among the 30 patients included in the study, 46 focal lesions were detected. Twenty-four lesions were metastatic lesions from primary cancer, 7 lesions were hepatocellular carcinoma (HCC), 9 lesions were hemangiomas, and 6 lesions were simple cysts. There was highly significant difference between the mean ADC of the malignant lesions (metastasis and HCC) and the mean ADC of benign lesions (hemangiomas and cysts). The ADC of malignant lesion was much less than that of benign lesion. The mean ADC of malignant lesions $(n=31)$ was $0.73 \pm 0.19 \times 10^{-3} \mathrm{~mm}^{2} / \mathrm{s}$, and the mean ADC of benign lesions $(n=15)$ was $1.94 \pm 0.68 \times 10^{-3} \mathrm{~mm}^{2} / \mathrm{s}(p$ value $<0.001)$. There was no significant difference between the cysts and hemangiomas. There was no statistically significant difference between the metastases and hepatocellular carcinoma.
\end{abstract}

Conclusion: ADCs values were able to differentiate benign from malignant lesions. ADC should be considered in the work up of patients with hepatic focal lesions.

Keywords: Liver, Diffusion, Hepatic focal lesions, Hepatocellular carcinoma

\section{Background}

Liver masses have currently been recognized and gained great attention. Most of these masses are known to be benign. Also, many cancer patients may coincidently have benign liver focal lesions that are confused for metastasis or primary liver malignancy [1]. The precise characterization of hepatic focal lesions is crucial for the diagnosis and management of patients [2], and accurate evaluation is essential to avoid unnecessary and incorrect intervention or treatment [1]. Common benign tumors include hemangiomas, fatty infiltration, adenoma, and cysts. As regards malignant

\footnotetext{
* Correspondence: shehadina@yahoo.com

${ }^{2}$ Department of Internal Medicine, Allergy and Clinical Immunology Unit, Ain Shams University, Khalifa El-Maamon St., Abbasiya sq, Cairo 11566, Egypt Full list of author information is available at the end of the article
}

liver tumors, metastases are considered 20 times more common than primary hepatocellular carcinoma [3].

Egypt is known for its high prevalence of hepatitis $\mathrm{C}$ virus [4], and therefore, close follow-up is essential to identify and detect the cases with hepatocellular carcinoma (HCC), which represents the fourth most common cancer in Egypt [5]. The serum biomarker, alpha-fetoprotein (AFP), is most commonly used in early diagnosis of HCC [6], However, some studies showed that AFP levels appeared to be normal in $40 \%$ of cases with HCC especially in their early stages $[7,8]$. Other studies demonstrated high levels of AFP in cirrhotic patients and patients with chronic hepatitis $[9,10]$. Usually confirmation of diagnosis of HCC is done by routine biopsy. Unfortunately, biopsy exposes 
the patient to undesirable risks and may delay diagnosis and treatment [11].

In the past few years, diagnosis of HCC through radiology has gained great support and attention. The international guidelines, European Association for the Study of the Liver (EASL) and American Association of the Study of Liver Disease (AASLD), accept and approve the use of radiology for diagnosis of HCC provided that it meets high-quality and defined imaging criteria [11].

The current imaging procedures to diagnose focal lesions are ultrasonography and computed tomography. Magnetic resonance imaging (MRI) is reserved to characterize lesions when not specified by other modalities. The inherent advantages of high contrast resolution, lack of ionizing radiation, as well as usage of advanced sequences and MR techniques renders it a favored modality [12].

The hepatic focal lesions are diagnosed based on their morphology and intensity on different sequences and their contrast behavior. There is still further accuracy needed for further differentiation between benign and malignant lesions [13].

Diffusion MRI has been used recently as a tool for further characterization of the hepatic focal lesions. This is available through measurement of apparent diffusion coefficient (ADC). Several studies have reported that ADC can contribute to the differentiation between benign and malignant lesions. The concomitant use with contrast enhancement criteria increases the accuracy of detection and characterization [14].

Therefore, the aim of the current work was to study a group of patients with hepatic focal lesions using the diffusion sequence as well as calculating the ADC value to differentiate between benign and malignant lesions.

\section{Methods}

Thirty patients with focal hepatic lesions were included in this study. The study was performed between June 2017 and January 2018. Patients with liver disease or known focal liver lesions who attended the Internal Medicine outpatient clinic or the emergency room at
Ain Shams University Hospital were admitted and referred to the MRI unit of Ain Shams University Hospital for completion of the study. Informed verbal consent was taken from the patients and the local ethical committee approved the study. Patients were diagnosed according to AASLD practice guidelines [15]. Biopsy was done for some cases (to confirm whether HCC or metastases) and imaging, including MRI with diffusion-weighted imaging (DWI), was done for participants.

\section{MRI examination}

Conventional MRI, post-Gd-DTPA dynamic and diffusion MR imaging were performed. First blind characterization and detection of focal lesions was performed, and then the diffusion images with ADC values were reviewed. All imaging results were verified against a standard of reference, which is a pathological verification. MR imaging was performed on high-field system (1.5 Tesla) magnet units (Philips Intera) using a phased array coil to cover the whole liver.

\section{MR protocol used \\ Pre-contrast imaging included}

Steps of pre-contrast imaging:

1. Axial T1-weighted imaging (T1WI)

2. Coronal T2-weighted imaging

3. Axial T2 SPAIR

4. Axial $\mathrm{T} 1$ in and out of phase

5. Axial heavy $\mathrm{T} 2$-weighted imaging

- T1-weighted (T1W) images: repetition time $(\mathrm{TR})=$ $10 \mathrm{~ms}$, echo time $(\mathrm{TE})=4.58 \mathrm{~ms}$, matrix 179/320, slice thickness 7-8 mm, slice gap 1-2 mm, and FOV $355 \mathrm{~mm}$.

- T2-weighted (T2W) images (single shot free breathing): $\mathrm{TR} \geq 445 \mathrm{~ms}$, $\mathrm{TE}=26-28 \mathrm{~ms}$, matrix $180-200 \times 240$ with a field of view: 365 , slice thickness 7-8 mm, slice gap 1-2 mm.

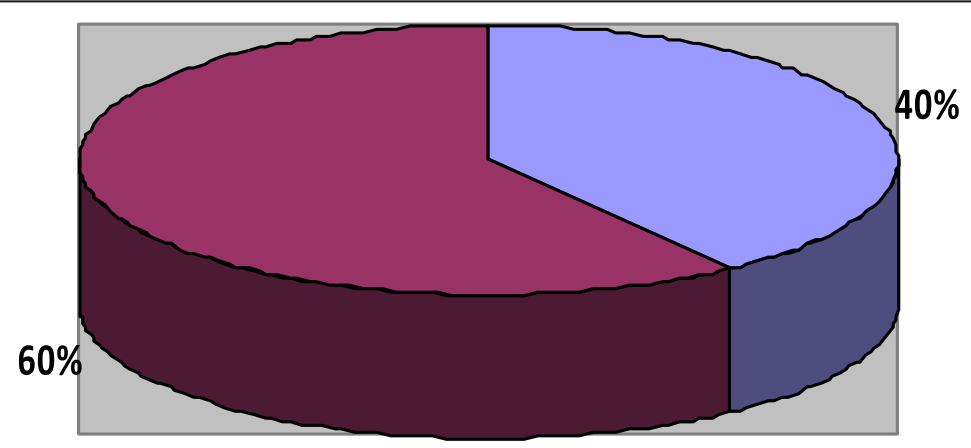

$\square$ male

female

Fig. 1 Gender distribution of patients 


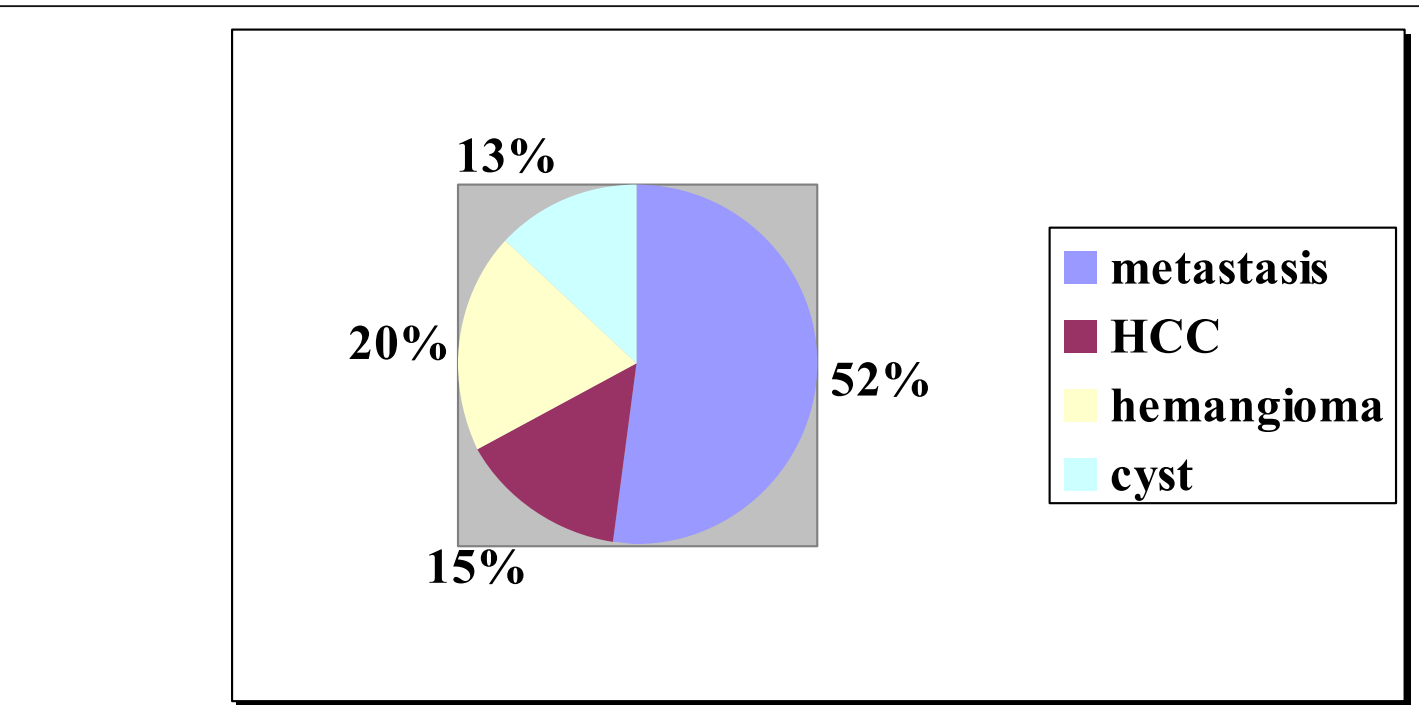

Fig. 2 Percentage of the lesions according to the pathological types

- T2 SPAIR (Spectral Attenuated Inversion Recovery) fat suppression sequence: $\mathrm{TR} \geq 400 \mathrm{~ms}, \mathrm{TE}=80 \mathrm{~ms}$, matrix $204 \times 384$ with a field of view: 365 slice thickness 7-8 mm, slice gap 1-2 mm.

- In phase and out phase gradient echo sequence (Dual/ FFE): TR $=75-100 \mathrm{~ms}$, TE $=4.6 \mathrm{~ms}$ for in phase and $2.3 \mathrm{~ms}$ for out phase, matrix $143 \times 240$ with a field of view: 345 slice thickness $7-8 \mathrm{~mm}$, slice gap $0 \mathrm{~mm}$.

- Heavy T2-weighted images: TR $=520$ ms, TE $=200$ ms, matrix 235/384 with a field of view: 375 slice thickness 7-8 mm, slice gap 1-2 mm.

\section{Dynamic study}

Dynamic study was performed after bolus injection of 0.1 $\mathrm{mmol} / \mathrm{kg}$ body weight of Gd-DTPA at a rate of $2 \mathrm{ml} / \mathrm{s}$, flushed with $20 \mathrm{ml}$ of sterile $0.9 \%$ saline solution from the antecubital vein. The injection of contrast media and saline solution was performed manually. Dynamic imaging using T1 THRIVE (high resolution isotropic volume Examination) technique was performed in triphasic way [arterial phase (16-20 s), porto-venous phase (45-60 s), and delayed equilibrium phase (3-5 $\mathrm{min}$ ) after administration of contrast media.

\section{Diffusion study}

Respiratory-triggered fat-suppressed single-shot echoplanar DW imaging was performed in the transverse plane with tri-directional diffusion gradients by using $b$ values 0,500 , and $1000 \mathrm{~s} / \mathrm{mm}^{2}$ to increase sensitivity to cellular packing. Parallel imaging with generalized auto-calibrating partially parallel acquisition (GRAPPA) with an acceleration factor of two was applied to improve image quality. The other parameters were as follows: repetition time (TR) $\geq 1880$ $\mathrm{ms}$, echo time $(\mathrm{TE})=70 \mathrm{~ms}$, number of excitations $(\mathrm{NEX})$
$=3$, matrix $256 \times 256$ with a field of view as small as possible with $52 \%$ rectangular field of view, slice thickness 7-8 mm, slice gap 1-2 $\mathrm{mm}$, scan time $3-4 \mathrm{~min}$.

\section{Imaging evaluation}

The morphological features of each lesion were recorded included size, shape, margin, signal characteristics, pattern of enhancement in the dynamic imaging, and as number and site of the detected focal lesions. Then provisional diagnosis was reported. A 10-year experienced radiologist (AY) reviewed the diffusion images with $\mathrm{ADC}$ values for final radiological characterization and detection of focal lesions.

\section{ADC calculation}

The mean ADC of each focal lesion detected was measured by drawing a region of interest over the lesion. The ADC was measured twice and the two measurements were averaged. To ensure that the same areas were measured, regions of interest were copied and pasted from DW images to ADC maps.

\section{The final diagnoses were reached according to standard of reference (SOR)}

For lesion characterization, all imaging results were refined against a predefined SOR. A range of procedures

Table 1 Number of lesions according to the size

\begin{tabular}{ll}
\hline Size of lesions $(\mathbf{c m})$ & Number of lesions \\
\hline $0.5-1$ & 8 \\
$1.1-2$ & 9 \\
$2.1-5$ & 21 \\
$5.1-10$ & 6 \\
$>10$ & 2 \\
\hline
\end{tabular}


Table 2 ADC of metastatic lesions and HCC

\begin{tabular}{|c|c|c|c|c|c|c|c|}
\hline \multirow{2}{*}{\multicolumn{2}{|c|}{ Sup-groups }} & \multicolumn{4}{|l|}{$A D C$} & \multicolumn{2}{|l|}{$T$ test } \\
\hline & & Min. & Max. & Mean & $\pm \mathrm{SD}$ & $T$ & $p$ value \\
\hline Metastasis & 24 & 0.20 & 0.90 & 0.70 & 0.18 & -1.427 & 0.164 \\
\hline $\mathrm{HCC}$ & 7 & 0.50 & 1.10 & 0.81 & 0.21 & & \\
\hline
\end{tabular}

was identified as valid "gold standards" for the characterization of lesions. The pathological results of the lesions were reviewed after blinded radiological diagnosis.

\section{Statistical analysis}

Data were analyzed using IBM $\odot$ SPSS $\odot$ Statistics version 23 (IBM@ Corp., Armonk, NY) and MedCalc® version 18.2.1 (MedCalc $\subset$ Software bvba, Ostend, Belgium).

Quantitative data were expressed as mean \pm standard deviation (SD). Qualitative data were expressed as frequency and percentage. Comparative analysis was done using Student's $t$ test and chi-square tests for quantitative and qualitative data, respectively. Correlations were done using Spearman's correlation coefficient. Probability ( $p$ value) was considered significant if it is $<0.05$, highly significant if $p<0.01$ and insignificant if $p>0.05$.

\section{Results}

Thirty patients were enrolled in this study. The age ranged between 18 years and 73 years with mean age of $48.5 \pm 18.37$ years (Fig. 1).
There were 46 focal hepatic lesions in our patients. Twenty-four lesions (52\%) were metastatic lesions from primary cancer, 7 lesions (15\%) were HCC, 9 lesions (20\%) were hemangiomas, and 6 lesions (13\%) were simple cystic (Fig. 2).

As regards the size of the lesions, 8 lesions were measured as $0.5-1 \mathrm{~cm}, 9$ lesions were measured as 1.1-2 $\mathrm{cm}, 21$ lesions were measured as $2.1-5 \mathrm{~cm}, 6$ lesions were measured as $5.1-10 \mathrm{~cm}$, and only 2 lesions were measured as more than $10 \mathrm{~cm}$ (Table 1 ).

All cystic lesions showed facilitated diffusion, where they showed reduction of signal intensity on increasing the $b$ values, and those which did not show reduction of signal showed high signal on ADC map, which also reflects facilitated diffusion. On the other hand, all solid lesions showed restricted diffusion evidenced by increased signal on increasing the $b$ values and low signal on ADC maps.

\section{$A D C$ values}

ADC values were obtained for all 46 focal hepatic lesions detected at consensus reading. The mean $\mathrm{ADC}$ value of the 24 metastatic lesions was $0.7 \pm 0.18 \times 10^{-3} \mathrm{~mm}^{2} / \mathrm{s}$. The mean $\mathrm{ADC}$ value of the 7 cirrhotic lesions in $\mathrm{HCC}$ patients was $0.81 \pm 0.21 \times 10^{-3} \mathrm{~mm}^{2} / \mathrm{s}$. The difference between the mean ADC of the two subtypes of malignant lesions was statistically insignificant ( $p$ value 0.16 , NS) (Table 2) (Figs. 3, 4 and 5).

As regards the ADC of benign lesions (hemangioma and cystic lesions), the mean ADC of hemangioma was

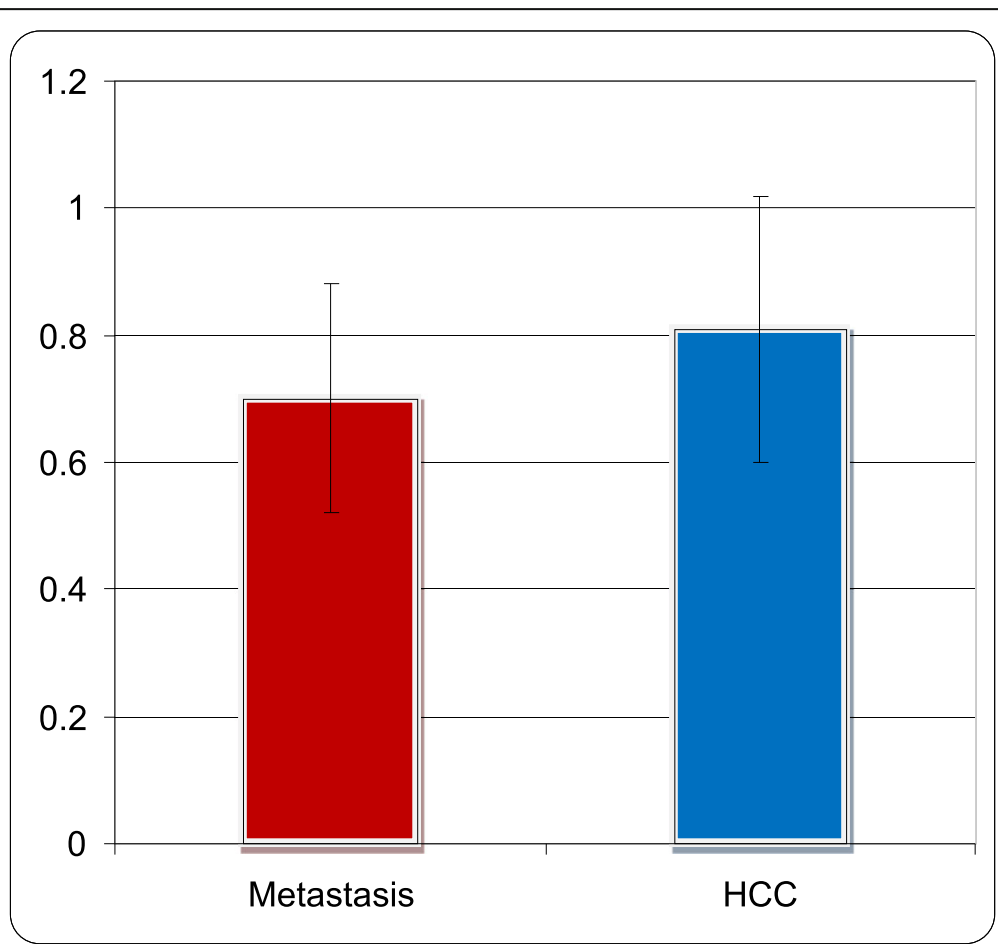

Fig. $3 \mathrm{ADC}$ of metastatic lesions and HCC 

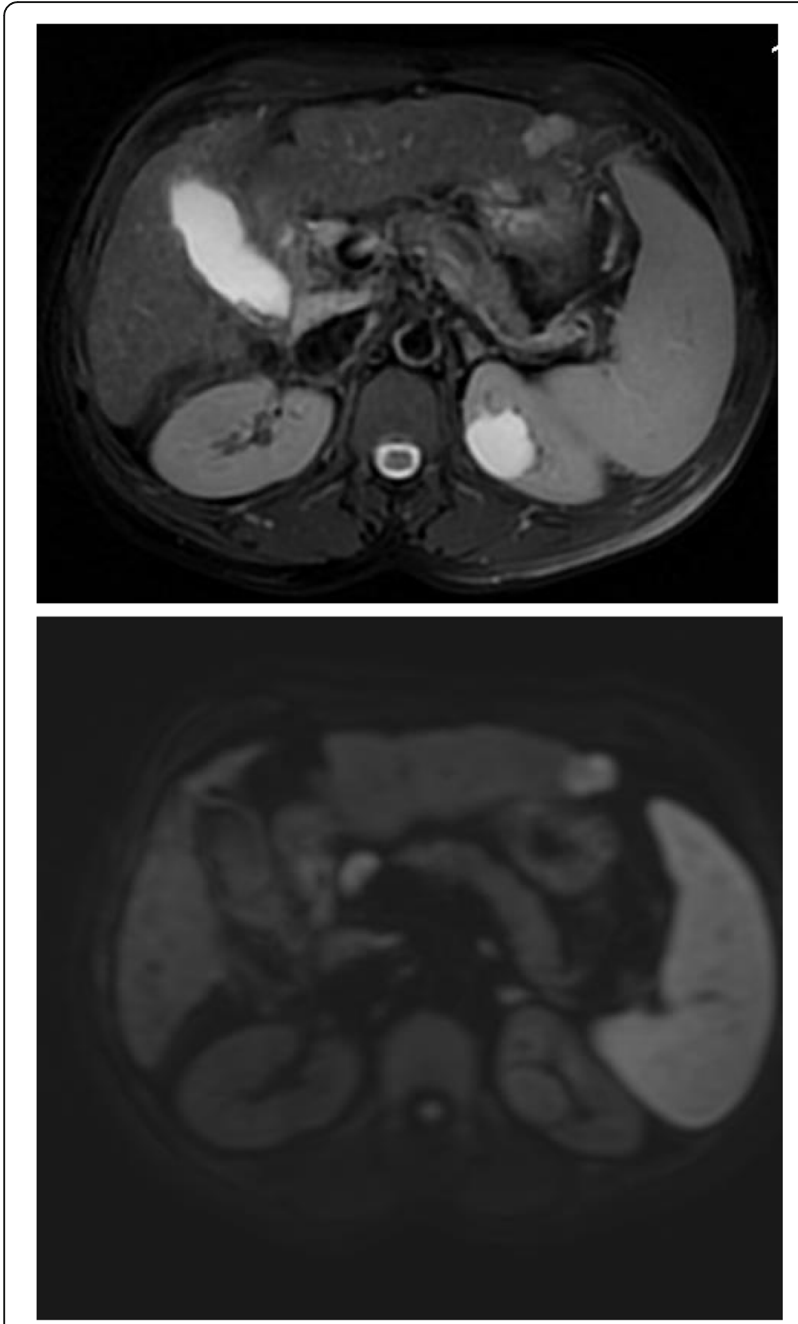

Fig. 4 a A 54-year-old male with liver cirrhosis and elevated Alfa feto protein proved to be hepatocellular carcinoma. Axial T2 fat sat shows a hyperintense focal lesion (arrowed). $\mathbf{b}$ Axial DWI, $b$ value $=$ 600 showing restricted diffusion within the lesion

$1.72 \pm 0.55 \times 10^{-3} \mathrm{~mm}^{2} / \mathrm{s}$, and the mean ADC of cystic lesions was $2.27 \pm 0.78 \times 10^{-3} \mathrm{~mm}^{2} / \mathrm{s}$. The difference between the mean ADC of the two subtypes of benign lesions was statistically insignificant ( $p$ value $0.13, \mathrm{NS}$ ) (Table 3) (Fig. 5).

There was a highly significant difference between the mean ADC of the malignant lesions (metastasis and HCC) and the mean $\mathrm{ADC}$ of benign lesions (hemangioma and cyst). The mean ADC of malignant lesions $(n=31)$ was $0.73 \pm 0.19 \times 10^{-3} \mathrm{~mm}^{2} / \mathrm{s}$ and the mean ADC of benign lesions $(n=15)$ was $1.94 \pm 0.68 \times 10^{-3} \mathrm{~mm}^{2} / \mathrm{s}$ ( $p$ value $<0.001$, S) (Table 4, Fig. 6). The ADC of malignant lesions was much less than that of benign lesions (Fig. 7).

\section{Discussion}

Focal lesions of the liver are a commonly encountered incidental finding. Focal lesions could be benign or
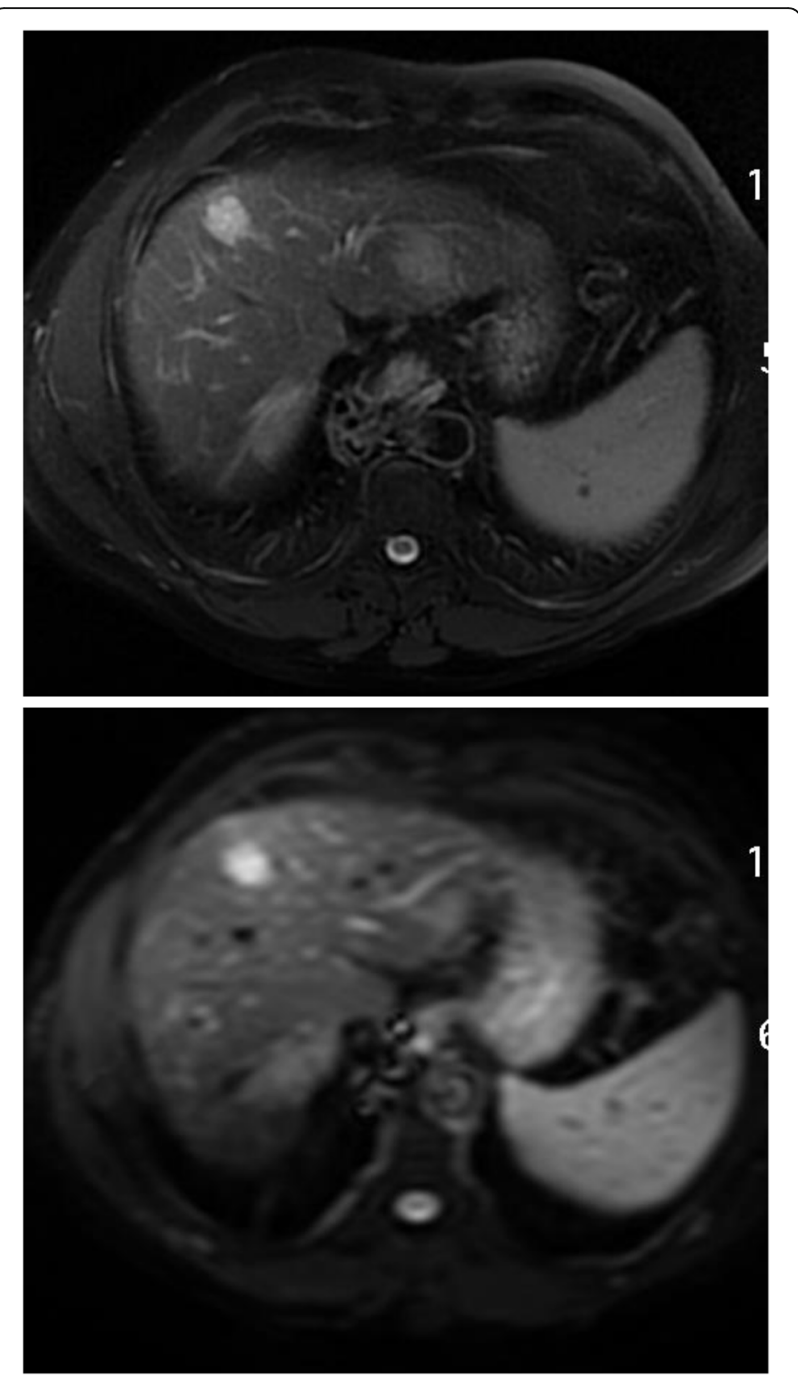

Fig. 5 a A 64-year-old male with history of pancreatic carcinoma. Axial T2 WI fat sat shows hyperintense focal lesion (arrowed). b Axial DWI $b$ value $=600$ showing restricted diffusion

malignant, representing a diagnostic dilemma, especially with the high prevalence of viral hepatitis in Egypt. Thus, early diagnosis is warranted to ensure optimal and timely management of hepatic focal lesions. Currently, the guidelines are dependent on ultrasonography and computed tomography; MR is reserved for further characterization since it has higher contrast resolution, safe in vulnerable groups of patients due to radiationfree technique and a safe contrast agent [16].

Table 3 ADC of hemangioma and cyst

\begin{tabular}{lllllllll}
\hline Sup-groups & & \multicolumn{2}{l}{ ADC } & & & & \multicolumn{2}{c}{$\boldsymbol{T}$ test } \\
\cline { 2 - 6 } \cline { 5 - 6 } & & Min. & Max. & Mean & $\mathbf{\pm S D}$ & & $\boldsymbol{T}$ & $\boldsymbol{p}$ value \\
\hline Hemangioma & 9 & 1.30 & 3.00 & 1.72 & 0.55 & -1.593 & 0.135 \\
CYST & 6 & 1.50 & 3.20 & 2.27 & 0.78 & & \\
\hline
\end{tabular}


Table 4 ADC of malignant and benign lesions

\begin{tabular}{lllllll}
\hline Groups & ADC & & & & \multicolumn{1}{c}{ test } & \\
\cline { 2 - 4 } \cline { 6 - 7 } & $\boldsymbol{N}$ & Mean & $\mathbf{\pm S D}$ & & $\boldsymbol{T}$ & $\boldsymbol{p}$ value \\
\hline Malignant & 31 & 0.73 & 0.19 & -9.279 & $<0.001$ \\
Benign & 15 & 1.94 & 0.68 & & \\
\hline
\end{tabular}

Diffusion imaging carries the advantage of using noncontrast agent, in addition to being dependent on tissue cellularity, organization, and integrity of cell membranes. These factors render it useful for detecting malignant lesions and differentiating tumorous from non-tumorous tissues [17].

In the current study, we aimed to demonstrate the use of diffusion MRI in differentiating benign from malignant liver lesions using the current sequence and comparing it to previously published literature. We used $3 b$ values 0,200 , and 800 as used in several studies including Demir et al. in 2007, Koike et al. in 2009, and Hosny et al. in 2010 [18-20].

In our study, the lesions were detected on DWI. Interestingly, the smallest detected lesion was $0.5 \mathrm{~cm}$, thus demonstrating its capability of detecting small lesions. While cysts and hemangiomas showed facilitated diffusion, solid neoplastic lesions showed restricted diffusion. This came in agreement with Taouli and Koh who reported that cellular hepatic lesions such as tumors demonstrated restricted diffusion at $b$ values higher than $500 \mathrm{~s} / \mathrm{mm}^{2}$ [17]. This could be explained by the fact that DWI depends on several factors including tissue cellularity. Since the main target of DWI is extracellular diffusion, high cellular tissue leads to a short diffusion path, resulting in low ADC values as seen in solid malignant lesions. On the other hand, low cellular lesions with less structural barriers as seen in cysts enhance longer diffusion path [21-23].

The absolute ADC values of different types of lesions were variable due to differences in techniques applied by $b$ values, different breath measurement techniques, and mathematical logarithms. Petra and Eric stated similar findings in 2010 [24]. The authors compared their findings with previous studies that used different DWI techniques, and reported that some publications preferred using breath-hold, or in other words respiratory triggered DWI, rather than using the conventional T2weighed MRI, while others favored both modalities in conjunction to obtain better results especially in atypical lesions, in order to avoid artifacts that might occur with measuring techniques of DWI.

The ADC measurement of benign and malignant lesions was significantly different with $p$ value $<0.0001$, which comes in agreement with a previous study by Onur et al. 2012. The authors found the mean ADC values differentiating benign and malignant lesions were statistically significant [25]. According to another study,

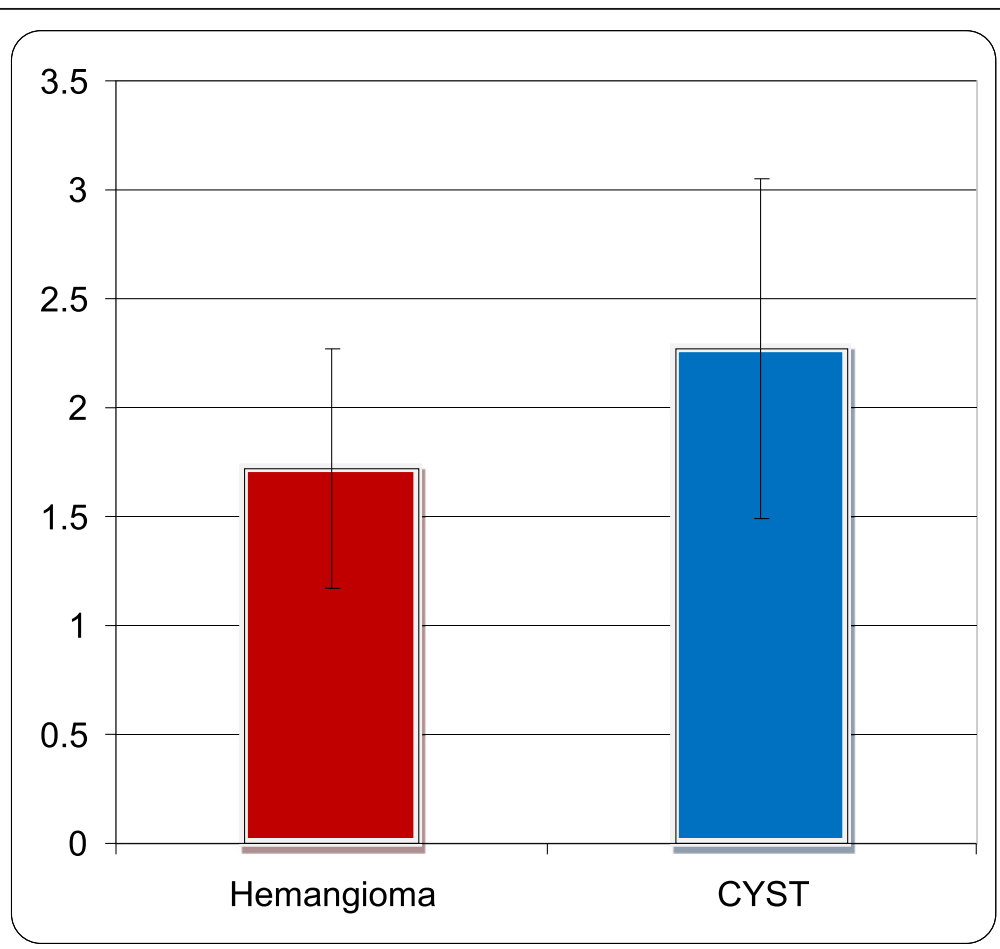

Fig. 6 ADC of hemangioma and cyst 


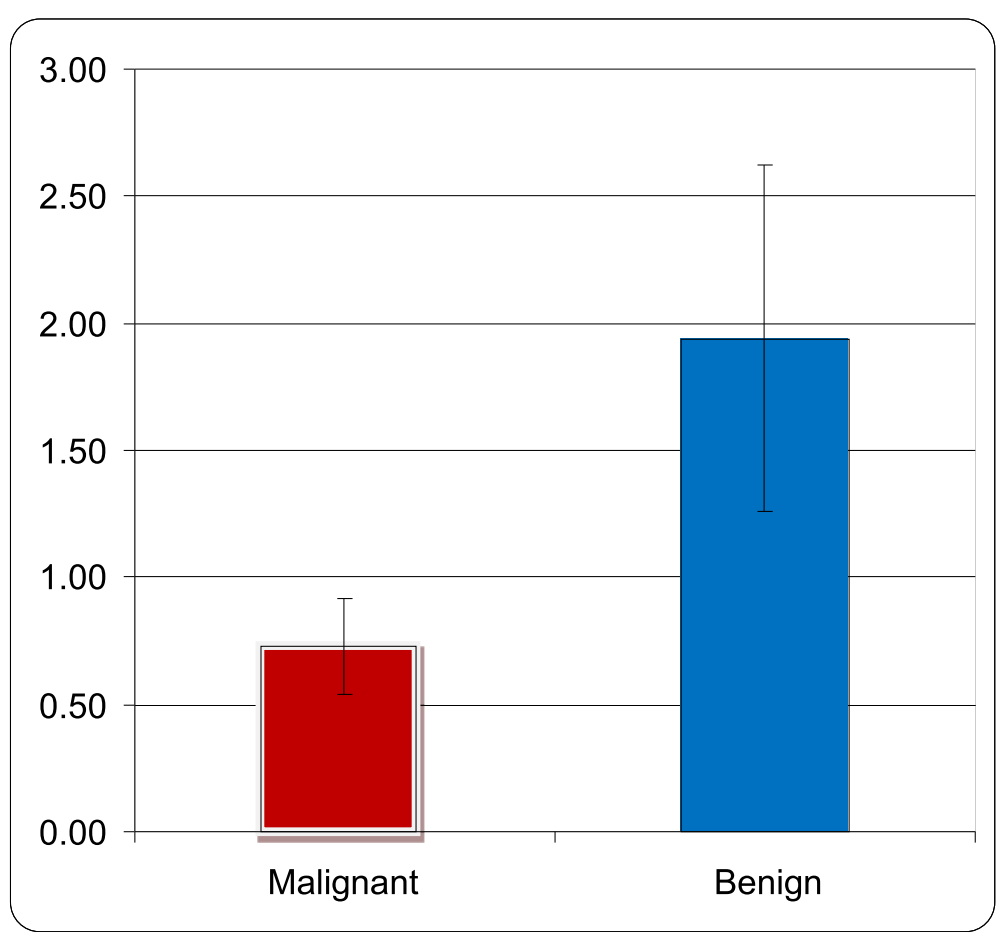

Fig. 7 ADC of malignant and benign lesions

although the authors recommended that the value of DWI should be highly considered, and showed the importance of ADC in focal lesions, they advised it careful interpretation since overlaps may occur between focal lesions as hemangiomas and metastases [26].

Some studies have indicated that AFP has substantially limited diagnostic accuracy in detecting small HCC [27]. As mentioned previously, AFP may be normal. We hence speculate that combination of AFP and ADC together could be useful in predicting malignancy. Unfortunately, they cannot differentiate primary from secondary malignancy.

Our study has some limitations. First, the DW data set was only respiratory triggered which has some limitations including cardiac motion artifacts and noise contamination that may distort ADC values. Second, the small number of patients included, especially in the subgroups of benign and malignant groups respectively. Additionally, benign lesions were limited to hemangiomas and cysts, while adenomas, focal nodular hyperplasia, and abscesses were not studied. Future multi-center studies with a larger sample size and including more pathologies of benign liver focal lesions are warranted to confirm our findings.

\section{Conclusion}

In conclusion, the DWI with ADC calculation could be used to differentiate between benign and malignant liver focal lesions. We recommend incurring such calculation in institutions across Egypt for accurate characterization of liver focal lesions. However, it is still not of clear value in identifying individual lesions; thus, we recommend further larger scale, multi-center studies to detect further modalities to identify individual lesions and differentiate between benign and malignant liver focal lesions.

\section{Abbreviations}

ADC: Apparent diffusion coefficient; AFP: Alpha-fetoprotein; AASLD: American Association of the study of liver disease; DWI: Diffusion-weighted imaging;

TE: Echo time; EASL: European association for the study of the liver; GRAPPA: Generalized auto-calibrating partially parallel acquisition; HCC: Hepatocellular carcinoma; MRI: Magnetic resonance imaging; NEX: Number of excitations; TR: Repetition time; SD: Standard deviation; SPSS: Statistical Program for Social Science; T1W: T1 weighted

\section{Acknowledgements}

We would like to acknowledge the contributions of the Public Health and community department for helping in the biostatistical analysis.

\section{Authors' contributions}

SA made substantial contributions to the design of the work. AY made substantial contributions to the conception of the idea and substantial revision of the work. AS made substantial contributions to acquisition and analysis of data. DS contributed to revising the work and interpretation, acquisition, and analysis of data. All authors have read and approved the final manuscript.

\section{Funding}

Nil

Availability of data and materials Not applicable 


\section{Ethics approval and consent to participate}

The study ethical approval was obtained from Faculty of Medicine, Ain Shams University ethical committee. Confidentiality of data was ensured by the study team. An informed verbal consent was taken from each participant in the study following the provision of an explanation of the study rationale and procedures and the ethical committee approved the verbal consent. Ethical committee reference number: FWA-0000-17585.

\section{Consent for publication}

Not applicable

\section{Competing interests}

The authors declare that they have no competing interests.

\section{Author details}

'Department of Radiodiagnosis, Ain Shams University, Khalifa El-Maamon St., Abbasiya sq, Cairo 11566, Egypt. ${ }^{2}$ Department of Internal Medicine, Allergy and Clinical Immunology Unit, Ain Shams University, Khalifa El-Maamon St. Abbasiya sq, Cairo 11566, Egypt.

Received: 18 May 2020 Accepted: 24 November 2020

Published online: 10 December 2020

\section{References}

1. Heiken JP (2007) Distinguishing benign from malignant liver tumours. Cancer Imaging. 7:S1-S14

2. Heslin MJ, Medina-Franco H, Parker M et al (2001) Colorectal hepatic metastases: resection, local ablation, and hepatic artery infusion pump are associated with prolonged survival. Arch Surg. 136(3):318-323

3. Xia Q, Feng Y, Wu C et al (2015) Differentiation between malignant and benign solitary lesions in the liver with FDG PET/CT: accuracy of age-related diagnostic standard. Journal of Cancer. 6:40-47

4. Doss W, Hermez J, Atta H, Jabbour J (2018) Towards a hepatitis-free Egypt: is this achievable? East Mediterr Health J. 24(7):609-610. https://doi.org/10. 26719/2018.24.7.609

5. Akinyemiju T, Abera S, Ahmed M, Alam N, Alemayohu MA, Allen C et al (2017) The burden of primary liver cancer and underlying etiologies from 1990 to 2015 at the global, regional, and national level. JAMA Oncol. 3: 1683-1691

6. Song PP, Feng $X$, Zhang $K$ et al (2013) Perspectives on using desycarboxyprothrombin (DCP) as a serum biomarker: facilitating early detection of hepatocellular carcinoma in China. Hepatobiliary Surg Nutr. 2 227-231

7. Daniele B, Bencivenga A, Megna AS et al (2004) Alpha-fetoprotein and ultrasonography screening for hepatocellular carcinoma. Gastroenterology. 127(Suppl 1):S108-S112

8. Marrero JA (2005) Screening tests for hepatocellular carcinoma. Clin Liver Dis. $9(235-251): \mathrm{vi}$

9. Di Bisceglie AM, Hoofnagle JH (1989) Elevations in serum alpha-fetoprotein levels in patients with chronic hepatitis B. Cancer. 64:2117-2120

10. Colli A, Fraquelli M, Casazza G et al (2006) Accuracy of ultrasonography, spiral CT, magnetic resonance, and alpha fetoprotein in diagnosing hepatocellular carcinoma: A systematic review. Am J Gastroenterol. 101: 513-523

11. Heuman DM, Gilles HS, Solomon C et al (2012) Should a radiological diagnosis of hepatocellular carcinoma be routinely confirmed by a biopsy? No. Eur J Intern Med. 23(1):37-39

12. Chandarana H, Block TK, Rosenkrantz AB et al (2011) Free-breathing radial 3D fat-suppressed $T 1$-weighted gradient echo sequence: a viable alternative for contrast-enhanced liver imaging in patients unable to suspend respiration. Invest Radiol. 46(10):648-653

13. Vergara ML, Fernández M, Pereira R (2010) Diffusion-weighted MRI characterization of solid liver lesions. Revista Chilena De Radiología. 16(1):510

14. Parikh T, Drew SJ, Lee VS et al (2008) Focal liver lesion detection and characterization with diffusion-weighted MR imaging: comparison with standard breath-hold T2-weighted imaging. Radiology. 246(3):812-822

15. Bruix J, Sherman M (2011) Management of hepatocellular carcinoma: An Update. AASLD Practice Guideline. Hepatology 53(3):1020-1022

16. Iwasaki $Y$, Arai K, Katayanagi S et al (2004) Biomarkers for neoplasmas in digestive organs. Gan To Kagaku Ryoho. 31(7):1015-1020
17. Taouli B, Koh DM (2010) Diffusion-weighted MR imaging of the liver. Radiology 254(1):47-66

18. Demir Ol, Obuz F, Sagul O et al (2007) Contribution of diffusion-weighted MRI to the differential diagnosis of hepatic masses. Diagn Interv Radiol. 13(2):81-86

19. Koike N, Cho O, Nasu K et al (2009) Role of diffusion-weighted magnetic resonance imaging in the differential diagnosis of focal hepatic lesions. World J Gastroenterol. 15(46):5805-5581

20. Hosny IA (2010) Diffusion MRI of focal liver lesions. Pakistan journal of Radiology 20:01-07

21. Charles-Edwards EM, deSouza NM (2006) Diffusion-weighted magnetic resonance imaging and its application to cancer. Cancer Imaging 6:135-143

22. Thoeny HC, De Keyzer F (2007) Extracranial applications of diffusionweighted magnetic resonance imaging. Eur Radiol 17:1385-1393

23. Kwee TC, Takahara T, Ochiai R et al (2008) Diffusion-weighted whole-body imaging with background body signal suppression (DWIBS): features and potential applications in oncology. Eur Radiol 18:1937-1952

24. Petra GK, Eric J (2010) Diffusion-weighted Imaging in the liver. World journal of gastroenterology 16:1567-1576

25. Onur MR, Çiçekçi M, Kayalı A et al (2012) The role of ADC measurement in differential diagnosis of focal hepatic lesions. Eur J Radiol. 81(3):e171-e176

26. Bruegel M, Holzapfel K, Gaa J et al (2008) Characterization of focal liver lesions by $\mathrm{ADC}$ measurements using a respiratory triggered diffusionweighted single-shot echo-planar MR imaging technique. Eur Radiol. 18(3): 477-485

27. Tateishi R, Yoshida H, Matsuyama $Y$ et al (2008) Diagnostic accuracy of tumor markers for hepatocellular carcinoma: A systematic review. Hepatol Int. 2:17-30

\section{Publisher's Note}

Springer Nature remains neutral with regard to jurisdictional claims in published maps and institutional affiliations.

\section{Submit your manuscript to a SpringerOpen ${ }^{\circ}$ journal and benefit from:}

- Convenient online submission

- Rigorous peer review

- Open access: articles freely available online

- High visibility within the field

- Retaining the copyright to your article

Submit your next manuscript at $>$ springeropen.com 